\title{
Actinomyces neuii infection following ovarian cystectomy
}

\author{
Jennifer Lueken, Benjamin Wilson, Mason Holbrook, Erin E. Medlin* \\ Department of OB/GYN \& Women's Health, University of Louisville School of Medicine, Louisville, KY, United States
}

Received: December 5, 2018

Accepted: January 31, $2018 \quad$ Online Published: February 5, 2018

DOI: $10.5430 /$ css.v4n1p6

URL: https://doi.org/10.5430/css.v4n1p6

\begin{abstract}
Background: Abdominal Actinomyces infections are rare complications of surgery. A case of Actinomyces neuii presenting as a severe postoperative pelvic infection is described.

Case: A 30 year-old nulligravid female was transferred from an outside hospital for a postoperative fluid collection, fever, leukocytosis, and abdominal pain following exploratory laparotomy and bilateral ovarian cystectomy. She rapidly decompensated requiring emergent exploratory laparotomy, bilateral salpingooophorectomy, and abdominal washout. Cultures isolated A. neuii. Following a prolonged ICU admission, she was treated with prolonged antibiotics and made a complete recovery.

Conclusions: Rare pathogens, such as A. neuii, may cause severe infections following gynecologic surgery. Multidisciplinary care at tertiary care facilities is critical.
\end{abstract}

Key Words: Ovarian cyst, Actinomyces, Sepsis, Intraabdominal infection

\section{INTRODUCTION}

Abdominal Actinomyces infections are rare. Actinomyces spp. are gram positive, filamentous anaerobic bacteria, characterized by pigment-producing rods forming filamentous branches. ${ }^{[1]}$ Actinomyces form a component of the intestinal commensal flora and vaginal flora, and are generally not pathogenic. However, infections occur through a breach in an anatomic barrier, leading to abscess formation, sinus tracts, or fistulas with a characteristic release of "sulphur granules" resulting in a pungent odor. ${ }^{[2]}$ Coinfection with other organisms is common and contributes to the pathogenesis. ${ }^{[1]}$ Actinomycosis, a chronic granulomatous disease, occurs most frequently in the orocervicofacial regions (50\%-60\%), but may also occur in the abdomen, thorax or in the pelvis related to intrauterine contraceptive devices (IUDs). ${ }^{[1,2]}$ The most common Actinomyces is Actinomyces israelii, but several others have been described through the use of biochemical assays. ${ }^{[3]}$

Actinomyces neuii, a species of Actinomyces, was first described in 1994 by Funke et al. ${ }^{[4,5]}$ In contrast to other species of Actinomyces, it is a diphtheroidal organism without branching, has both anaerobic and aerobic growth, and has greater than 60 strains. $^{[5]}$ There are limited reports of a classic Actinomyces presentation with gram-positive filaments and sulphur granules. ${ }^{[5]}$ The diagnosis of $A$. neuii is based on 16S rRNA sequencing. A. neuii is known to be endogenous to the human body. Previous case studies have reported infections and abscesses caused by $A$. neuii in the skin, eyes, heart, pericardium, bone, and placenta. ${ }^{[5]}$ However, there have been few reports of $A$. neuii as the causative agent in intra-abdominal infections following pelvic surgery. The case presented here of a dramatic intraabdominal $A$.

${ }^{*}$ Correspondence: Erin E. Medlin; Email: e0med101@ louisville.edu; Address: Department of OB/GYN \& Women's Health, University of Louisville School of Medicine, Louisville, KY, United States. 
neuii infection highlights a new area of pathogenesis for this species.

\section{Case presentation}

A 30-year-old obese nulliparous female with past medical history significant only for non-insulin dependent diabetes mellitus was transferred from an outside hospital in septic shock with pelvic and incisional abscesses. She had an attempted laparoscopic bilateral ovarian cystectomy at an outside hospital that was converted to laparotomy via Pfannenstiel skin incision due to concern for cystic rupture 11 days prior to transfer for presumed dermoid ovarian cysts. She did not receive perioperative antibiotics as these were not indicated for this procedure. She represented on postoperative day 5 with complaint of nausea, vomiting, and fevers at home. Computed tomography (CT) scan demonstrated a $10 \mathrm{~cm} \times 11 \mathrm{~cm}$ abscess of the left adnexa, moderate ascites, dilated small bowel loops, and a $3 \mathrm{~cm} \times 11 \mathrm{~cm}$ fluid and gas containing collection within the anterior abdominal wall. She was started on vancomycin, levofloxacin, and cefoxitin. The Pfannenstiel incisional abscess spontaneously drained and culture grew out Staphylococcus. aureus and she was subsequently transitioned to gentamycin and clindamycin based on sensitivity results. While the patient remained afebrile, she had worsening leukocytosis with white blood cell count (WBC) of $29 \times 10^{9} / \mathrm{L}$ and developed significant hyponatremia to $123 \mathrm{mEq} / \mathrm{L}$, acute kidney injury, and lactic acidosis. She was transferred to a university tertiary care center given concern for developing sepsis (see Figure 1).

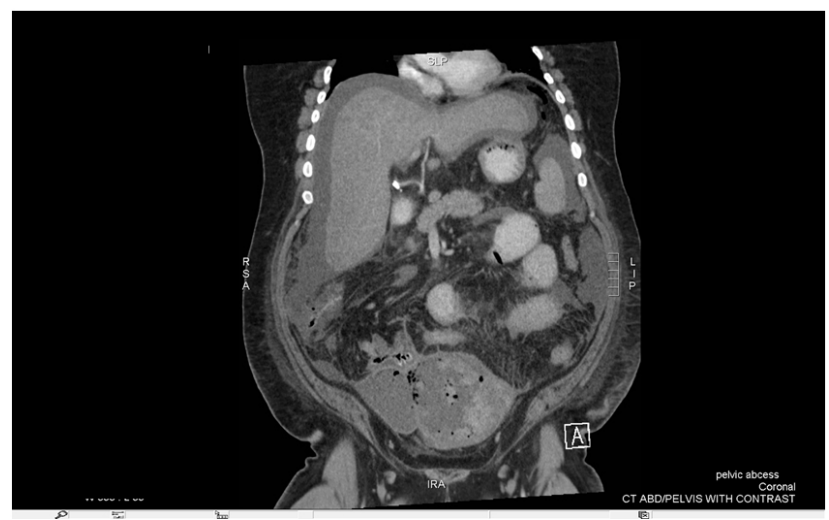

Figure 1. CT imaging of abdomen and pelvis of pelvic abscess

On arrival, patient was in septic shock and subsequently admitted to the ICU, requiring intubation for profound tachypnea and subsequent acute respiratory failure. Antibiotic coverage was broadened to meropenem, vancomycin, and clindamycin and patient was emergently taken to the operating room for exploratory laparotomy with drainage of pelvic abscess. Surgical findings were significant for a 1 $\mathrm{cm}$ defect of the Pfannenstiel incision with purulent fluid throughout subcutaneous tissue extending down to the fascia. With opening of the fascia, approximately 2,500 cc of purulent intra-peritoneal fluid with approximately 1,000 cc of necrotic/black fluid was encountered immediately on entry into the peritoneal cavity, as well as a $10 \mathrm{~cm} \mathrm{left}$ ovarian abscess with significant necrotic tissue and a $5 \mathrm{~cm}$ right ovarian abscess. Patient underwent a bilateral salpingooophorectomy and abdomino-pelvic washout with temporary abdominal closure. Abdomen had to be re-explored at bedside in the ICU the following day with further debridement of subcutaneous and fascial tissue and partial omentectomy for worsening septic shock unresponsive to maximum doses of norepinephrine, phenylephrine, and vasopressin and resulting organ system failure. Continuous peritoneal irrigation was implemented. Following bedside intra-abdominal reexploration with debridement, significant clinical improvement was noted and vasopressors were weaned.

Preliminary wound cultures were positive for $A$. neuii on hospital day 4 and infectious disease service was consulted given unusual pathogen with recommendation for long-term penicillin and clindamycin. Patient therefore underwent penicillin desensitization due to penicillin allergy. Repeated abdominopelvic washouts were done every other day with final abdominal closure with secondary fascial mesh closure on hospital day 8 . She was subsequently able to be extubated and transitioned from critical care. She was stable for discharge to acute rehab facility on hospital day 19 and did well following hospitalization with no wound issues at her 6 week postoperative visit. She was transitioned to oral amoxicillinclavulanate after completion of 6 weeks of IV penicillin and clindamycin with plan for 6 months of total antibiotic treatment. Final pathology from initial exploratory laparotomy with bilateral salpingo-oophorectomy and abdominopelvic washout demonstrated a benign Brenner tumor and multiloculated cystadenoma of the left ovary with torsion and diffuse acute inflammation and hemorrhage, pyosalpinx of the left fallopian tube, and multiloculated cystadenoma of the right ovary with diffuse acute inflammation and hemorrhage.

\section{Discussion}

This case reports a rare infectious complication following pelvic surgery. Surgical site infections following pelvic surgery are relatively uncommon with an incidence of $2.7 \%$ for superficial, deep, and organ space infections following hysterectomy. ${ }^{[6]}$ This is in large part due to the widespread implementation of prophylactic antibiotics prior to hysterectomy, where an infection rate of $33 \%$ was previously reported. ${ }^{[6]}$ In this case, preoperative antibiotics were not 
indicated per American College of Obstetricians and Gynecologists guidelines. ${ }^{[7]}$

Actinomyces infections are rare complications of surgical procedures, and are more commonly associated with disruption of the oropharynx or disruption of the bowel. ${ }^{[2]}$ Pelvic infections with Actinomyces most commonly arise from the appendix, however ascending infections from the intrauterine devices have been widely described. ${ }^{[8]}$ The incidence of Actinomyces identified on Pap smears of women using IUDs range from $0 \%-31 \%$, although this does not indicate or predict abscess formation. Actinomyces israelii is the most common isolate in pelvic infections associated with IUDs, and may result in tubo-ovarian abscesses. However, this patient had no history of IUD use or insertion. The species of Actinomyces isolated in this case, A. neuii, is unique in both its morphology and growth under aerobic conditions when compared to other species of Actinomyces. Infections described with this species include skin infection, endophthalmitis, bacteremia, endocarditis, mammary abscess, axillary abscess, and inguinal abscesses. ${ }^{[2]}$ Intraabdominal $A$. neuii is extremely rare, with only one case identified in the literature. Vanoeteran et al described a case of A. neuii intraabdominal infection following a cholecystectomy. ${ }^{[2]} \mathrm{Sim}-$ ilar to our case, this patient received a prolonged course of intravenous penicillin followed by oral antibiotics treatment for six months. This is the first case of A. neuii that has been identified following gynecologic surgery.

In this case, a severe infection with A. neuii developed. Koo et al. attempted to identify preoperative factors that may predict the severity of pelvic Actinomyces infection. The most common risk factors for pelvic Actinomyces infections were history of endometrial curettage, abortion, or intrauterine device use. ${ }^{[9]}$ Patients with aggressive Actinomyces infec- tions had a significantly higher incidence of fever, neutrophil percentage and white blood cell/hemoglobin ratio. Immunosuppression may contribute to worsening disease state. This patient had a severe infection resulting in septic shock, and presented with high fever and high neutrophil percentages. However, she was immunocompetent. No predisposing factors were identified.

In summary, this is a rare case of A. neuii isolated from an abdominopelvic abscess following gynecologic surgery. This rare infection may present with pelvic fluid collections, septic shock, and multi-organ system failure. Definitive diagnosis is made by bioassays including $16 \mathrm{~S}$ rRNA in additional to histochemical and microscopic examinations. Sulphur granules further support the diagnosis. Management requires consultation with critical care and infectious disease specialist to manage septic shock and long-term intravenous and oral antibiotics. Referral to a tertiary care center is required for comprehensive management. A full recovery is possible with multidisciplinary care and astute clinicians and laboratory personnel who identify this organism and care for the patient aggressively.

\section{Patient Consent}

The patient presented in this case report provided written consent for publication of this case report. Consent is available upon request.

\section{IRB APPROVAL}

This case report was considered exempt by our institutional IRB.

\section{CONFlicts OF INTEREST Disclosure}

The authors declare they have no conflicts of interest.

\section{REFERENCES}

[1] Bonnefond S, Catroux M, Melenotte C, et al. Clinical features of actinomycosis: A retrospective, multicenter study of 28 cases of miscellaneous presentations. Medicine (Baltimore). 2016; 95(24) e3923. PMid: 27311002. https ://doi .org/10.1097/MD.00000 00000003923

[2] Vanoeteren X, Devreese K, De Munter P. Abdominal actinomycosis: a rare complication after cholecystectomy. Acta Clin Belg. 2014; 69(2): 152-6. PMid: 24724763. https://doi.org/10.1179/00 $01551214 \mathrm{Z} .00000000034$

[3] Hall V. Actinomyces-gathering evidence of human colonization and infection. Anaerobe. 2008; 14(1): 1-7. PMid: 18222714. https: //doi.org/10.1016/j.anaerobe.2007.12.001

[4] Funke G, Stubbs S, von Graevenitz A, et al. Assignment of humanderived CDC group 1 coryneform bacteria and CDC group 1-like coryneform bacteria to the genus Actinomyces as Actinomyces neuii subsp. neuii sp. nov., subsp. nov., and Actinomyces neuii subsp. anitratus subsp. nov. Int J Syst Bacteriol. 1994; 44(1): 167-71. PMid: 8123558. https://doi.org/10.1099/00207713-44-1-167

[5] von Graevenitz A. Actinomyces neuii: review of an unusual infectious agent. Infection. 2011; 39(2): 97-100. PMid: 21340579. https://doi.org/10.1007/s15010-011-0088-6

[6] Lachiewicz MP, Moulton LJ, Jaiyeoba O. Pelvic surgical site infections in gynecologic surgery. Infect Dis Obstet Gynecol. 2015; 2015 : 614950. PMid: 25788822. https ://doi.org/10.1155/2015/6 14950

[7] Bulletins-Gynecology ACoP. ACOG practice bulletin No. 104: antibiotic prophylaxis for gynecologic procedures. Obstet Gynecol. 2009; 113(5): 1180-9. PMid: 19384149. https://doi.org/10.1 097/A0G.0b013e3181a6d011

[8] Westhoff C. IUDs and colonization or infection with Actinomyces. 
Contraception. 2007; 75(6 Suppl): S48-50. PMid: 17531616. https : //doi.org/10.1016/j. contraception.2007.01.006

[9] Koo YJ, Kwon YS, Shim JU, et al. Predictors associated with severity of pelvic actinomycosis. J Obstet Gynaecol Res. 2011; 37(12): 17926. PMid: 21794000 . https://doi .org/10.1111/j.1447-0756. 2011.01608. $\mathrm{x}$ 